\title{
THEORETICAL MODELS OF DIFFUSE CLOUDS
}

\author{
The Importance of Density Inhomogeneities and $\mathrm{H}_{2}$ Fluorescence
}

\author{
MARCO SPAANS \\ Johns Hopkins University \\ 3400 N. Charles St. Baltimore, MD21218, USA
}

\begin{abstract}
Two-dimensional model calculations for inhomogeneous diffuse and translucent clouds are presented which include line and continuum radiative transfer, thermal balance and chemical equilibrium. The effects of an inhomogeous density distribution on the abundances of chemical species and line maps are discussed. Additional calculations are presented for the fluorescently pumped $\mathrm{H}_{2}$ visual and infrared lines which emanate from spherical diffuse clouds. The results indicate that these lines yield sensitive probes of the ambient cloud density when combined with co-spatial HI observations.
\end{abstract}

\section{Introduction}

Diffuse and translucent clouds have been studied extensively for the past twenty years (Black \& Dalgarno 1977, van Dishoeck \& Black 1986 and references therein). With their moderate extinctions $\left(A_{\mathrm{V}} \approx 1-5 \mathrm{mag}\right)$ and low densities $\left(n_{\mathrm{H}} \sim 30-500 \mathrm{~cm}^{-3}\right)$, they are easily observable with the use of absorption line techniques. These clouds are generally illuminated by the average interstellar radiation of the Galaxy and their chemistry and thermal balance are directly linked to the impinging radiation field and ambient density. As such, they aid in our understanding of ion-molecule chemistry in interstellar space as well as the physical structure of the galactic interstellar medium.

Many comprehensive data sets exist for diffuse clouds along lines of sight toward bright stars (see van Dishoeck \& Black 1986 for an overview). These observations provide strong constraints on the pertaining density, temperature and illuminating radiation field. The aim of this contribution is to compare current theoretical models with existing measurements as well as new observations which have recently become available (see Meyer 
and Lucas \& Liszt in this volume). The emphasis will lie on the density structure of diffuse and translucent clouds and the influence of geometry on the abundance and (fluorescent) excitation of $\mathrm{H}_{2}$ and other atomic and molecular species like $\mathrm{C}, \mathrm{C}^{+}, \mathrm{CO}, \mathrm{OH}, \mathrm{CH}, \mathrm{HCO}^{+}$and $\mathrm{CH}^{+}$.

Nowadays a large (and growing) amount of data has been gathered in different molecular lines which indicate a highly inhomogeneous structure of interstellar clouds on different scales (Falgarone \& Pérault 1988; Marscher et al. 1993 and references therein). In order to interpret these observations, the inhomogeneous density structure needs to be taken into account in the models. Only then is it possible to determine the influence of the inhomogeneous structure on the abundances and excitation of the different species, and to infer the underlying density distribution (Stutzki et al. 1988; Tauber \& Goldsmith 1990; Howe et al. 1991; Meixner \& Tielens 1993).

The fluorescent excitation of $\mathrm{H}_{2}$ has also been studied extensively in the past two decades (Black \& Dalgarno 1977; Black \& van Dishoeck 1987; Sternberg 1988). These investigations have concentrated on plane-parallel slabs with large extinctions, so that all the impinging ultraviolet radiation is absorbed. The resulting intensities in the fluorescent lines are expected to be different for spherical diffuse clouds in which only part of the ultraviolet flux is absorbed. Also, recent advances in detector technology have made it possible to observe very weak lines in the infrared around $2 \mu \mathrm{m}$ and in the visual around $8000 \AA$. These effects are further investigated here for an ensemble of diffuse clouds covering a range in extinction, density and radiation field and are compared with observations.

\section{Model Description}

\subsection{INHOMOGENEOUS CLOUDS}

The details of the adopted method are described in Spaans (1996). Here a brief overview will be given. The continuum and line radiative transfer are computed with a Monte Carlo method. The self-shielding of $\mathrm{H}_{2}$ and $\mathrm{CO}$ and the shielding of the latter by the former are included in the one line approximation. The chemical and thermal equilibrium are solved simultaneously to within a percent. The dominant heating processes include heating by 1) Photo-electric emission from dust grains and PAHs; 2) cosmic rays; 3) $\mathrm{H}_{2}$ photodissociation, FUV pumping and formation; and 4) neutral carbon ionization. The bulk of the cooling is provided by 1) fine-structure line emission from $\mathrm{C}, \mathrm{C}^{+}$and $\mathrm{O}$; and 2) rotational line emission of $\mathrm{CO}$. The level populations are determined in statistical equilibrium (up to $J=20$ for $\mathrm{CO}$ ) and optical depth effects in the cooling lines are explicitly taken into account.

The chemical network includes 628 reactions between 63 species con- 
sisting of the elements $\mathrm{H}, \mathrm{He}, \mathrm{D},{ }^{12} \mathrm{C},{ }^{13} \mathrm{C},{ }^{16} \mathrm{O},{ }^{18} \mathrm{O}, \mathrm{N}, \mathrm{S}, \mathrm{Si}, \mathrm{Fe}, \mathrm{Mg}$, $\mathrm{Na}$ and $\mathrm{Ca}$. The inhomogenous density distribution is represented by a two-phase medium and is uniquely determined by values for the average density $\left\langle n_{\mathrm{H}}\right\rangle$, clump-interclump density ratio $R$, clump filling factor $F$ and clump size $\ell_{\mathrm{c}}$. The density field is constructed by randomly choosing sites for the putative clumps conform the numerical values of the parameters given above.

\subsection{SPHERICAL CLOUDS}

In Neufeld \& Spaans (1996) a detailed description of the numerical algorithm is provided. Here only the essentials are described. The clouds in question are assumed to be spherically symmetric and illuminated isotropically. Along 26 rays the incident ultraviolet flux is transported and attenuated by dust and $\mathrm{H}_{2}$ molecules. This procedure is iterated until the $\mathrm{H}_{2}$ abundance and the statistical equilibrium of the level populations changes by less than a percent.

The first 12 vibrational levels have been included in the equations of statistical equilibrium using the excitation energies and Einstein $A$ coefficients of Dabrowski (1984) and Turner, Kirby-Docken \& Dalgarno (1977), respectively. The adopted collisional de-excitation rates of $\mathrm{H}_{2}$ by $\mathrm{H}$ were based on the results of Green \& Truhlar (1979). Radiative pumping by ultraviolet radiation of all the Lyman and Werner band transitions was included for states of rotational quantum number $J \leq 14$. The ortho $J=1 /$ para $J=0$ ratio was fixed at a temperature-dependent value of $9 \mathrm{e}^{-170.485 / T}$. The resulting line intensity follows from an integration through the middle of the cloud and the attenuation resulting from dust in the visual and infrared is taken into account.

\section{Results}

\subsection{CHEMICAL ABUNDANCES AND LINE MAPS}

Figure 1 presents the results for an inhomogeneous slab illuminated isotropically from one side with $\left\langle n_{\mathrm{H}}\right\rangle=10^{3} \mathrm{~cm}^{-3}, R=10, F=25 \%$ and $\ell_{\mathrm{c}}=0.2 \mathrm{pc}$. The corresponding line maps for [CII] $158 \mu \mathrm{m}$ and [CI] $609 \mu \mathrm{m}$ emission are shown in Figure 2. It is apparent that neutral and ionized carbon are present over a much more extended region compared to the homogenous model as is further evidenced in the one-dimensional cuts depicted in Figure 3. This is caused by the enhanced penetration of ultraviolet photons through the low-opacity interclump medium.

The ionized and neutral carbon are located mostly in the interclump medium and on the surfaces of the clumps, respectively. The $\mathrm{CO}$ abundance 

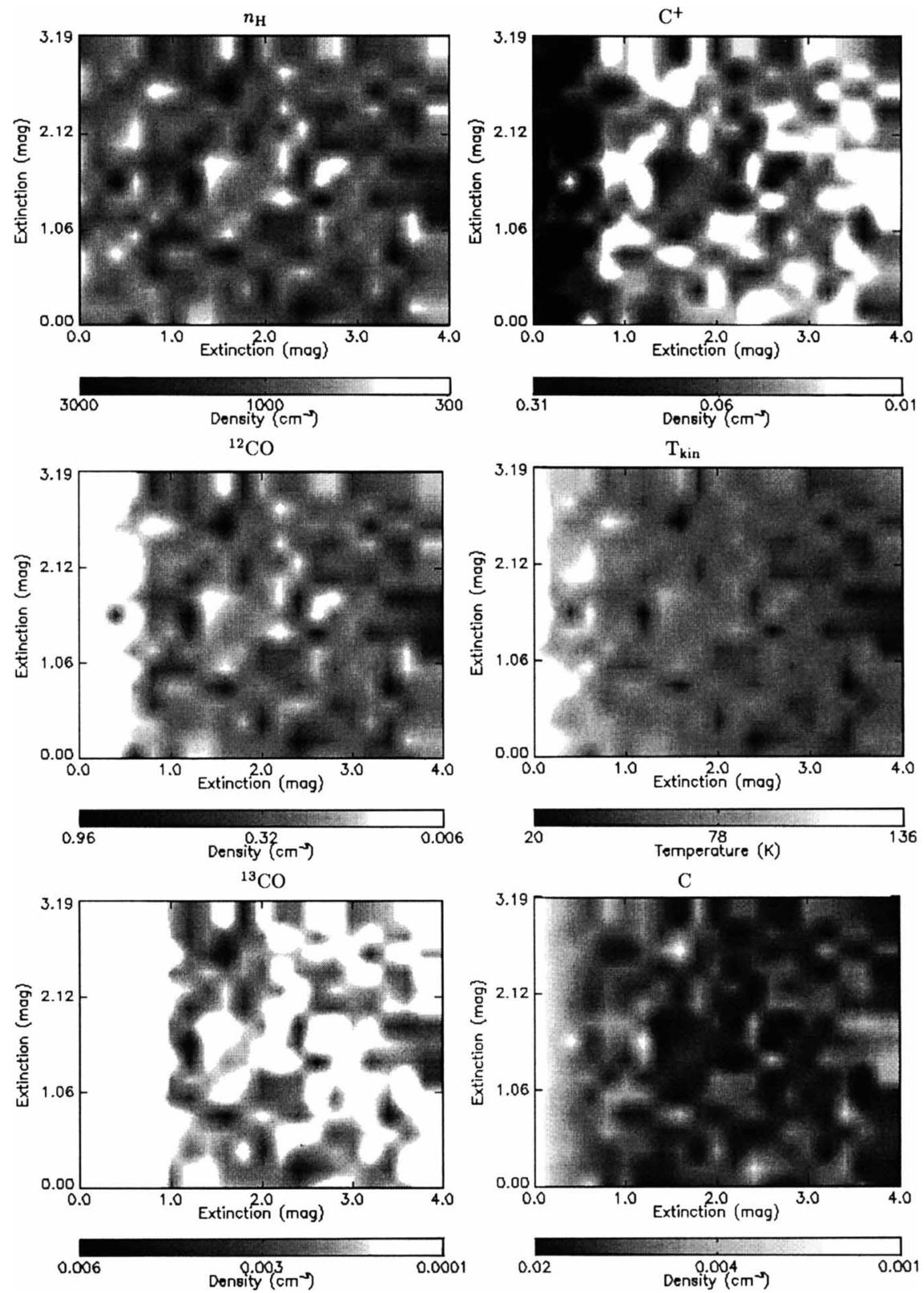

Figure 1. Maps of the $n_{\mathrm{H}}$ (upper left), $\mathrm{C}^{+}$(upper right), ${ }^{12} \mathrm{CO}$ (middle left), ${ }^{13} \mathrm{CO}$ (lower left), and $\mathrm{C}$ (lower right) density together with the temperature (middle right) for a generic model with $I_{\mathrm{UV}}=10, A_{\mathrm{V}}=4 \mathrm{mag},\left\langle n_{\mathrm{H}}\right\rangle=10^{3} \mathrm{~cm}^{-3}$, and a clump volume filling factor of $25 \%$. The clump-interclump density ratio $R=10$ and the clump size is $0.2 \mathrm{pc}$. 
[C] $609 \mu \mathrm{m}$

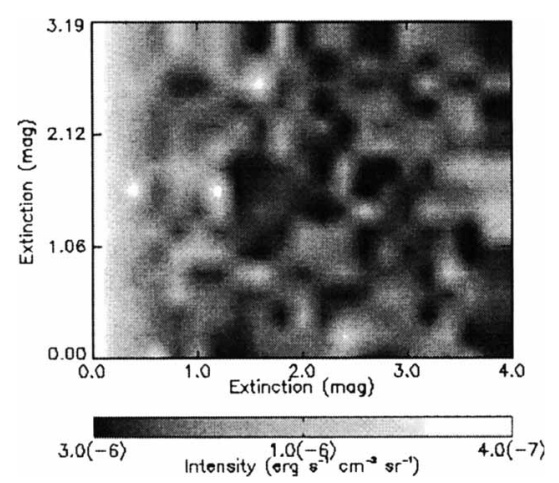

[CII] $158 \mu \mathrm{m}$

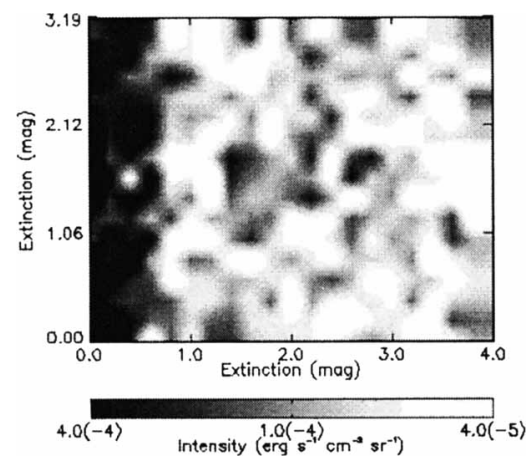

Figure 2. [CI] $609 \mu \mathrm{m}$ (upper panel) and [CII] $158 \mu \mathrm{m}$ (lower panel) line maps for the model of Figure 1.

is enhanced in the clumps and benefits from a narrower self-shielding $\mathrm{C}^{+}-\mathrm{C}-$ $\mathrm{CO}$ transition in these high density regions despite the stronger interclump ultraviolet radiation field.

\section{2. $\mathrm{H}_{2}$ FLUORESCENCE}

Because $15 \%$ of the absorptions in the Lyman and Werner systems connect to the continuum, photodissociation and ultraviolet pumping of $\mathrm{H}_{2}$ are intimately linked. In static equilibrium, the number of $\mathrm{H}_{2}$ molecules formed is equal to the number of $\mathrm{H}_{2}$ molecules dissociated which is a direct measure of the number of photons produced in some fluorescent line when 

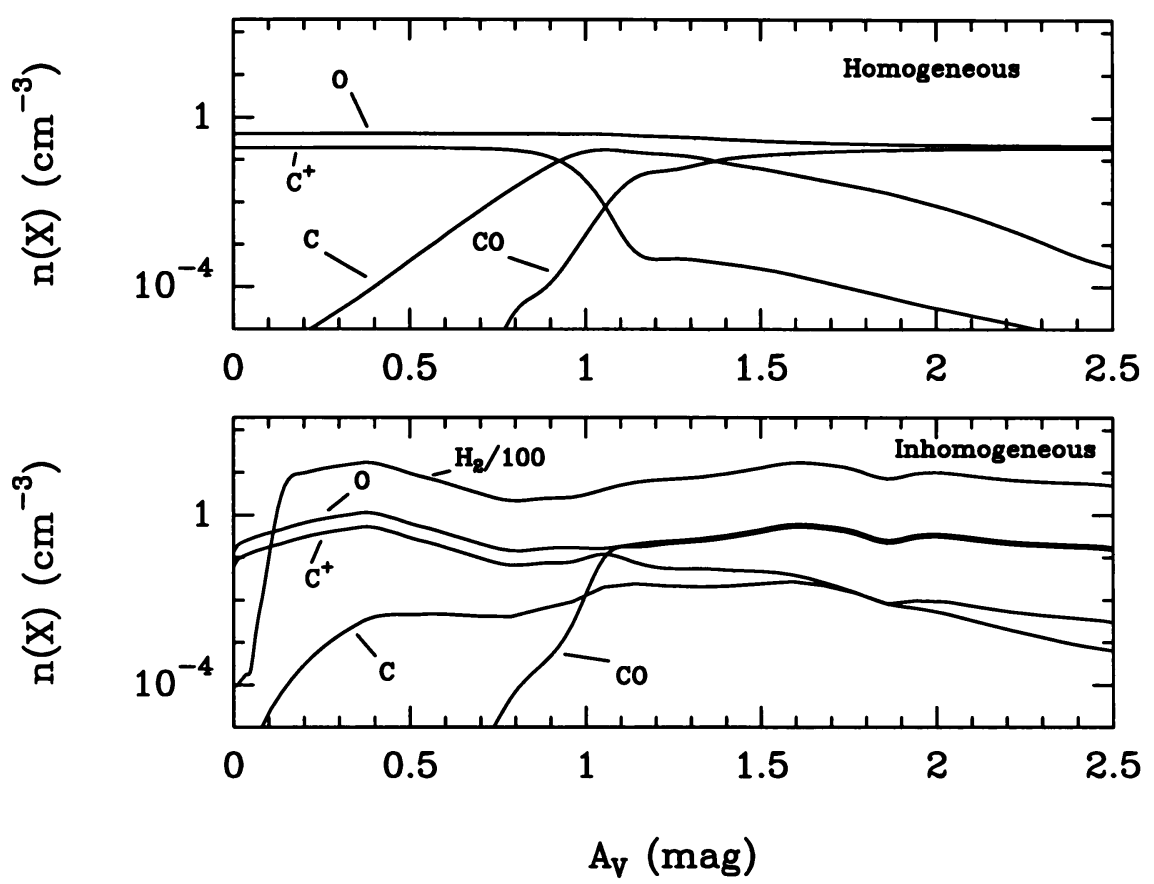

Figure 3. (top panel) Ionized to neutral carbon transition in a homogeneous cloud with $n_{\mathrm{H}}=10^{3} \mathrm{~cm}^{-3}$ and $I_{\mathrm{UV}}=10$. (lower panel) One-dimensional cuts through the corresponding inhomogenous model of Figures 1 and 2.

combined with the cascade branching ratios. That is, $\int R_{\text {form }}(T) n_{H} n(H)=$ $\int R_{\text {dis }} n\left(H_{2}\right) \propto I_{\text {line }}$, yielding a direct proportionality between line intensity, density and atomic hydrogen column density.

Figure 4 presents computations for isotropically illuminated spherical clouds for a range in density and extinction, and a value for the enhancement of the average interstellar radiation field $I_{\mathrm{UV}}=1$. The results are plotted as functions of the observable line intensity and atomic hydrogen column density. The scaling with density is evident. The turnover results when the extinction has become high enough to absorb the fluorescent emission from the backside of the cloud with respect to the observer. Additional calculations show that the fluorescent line intensities as functions of $N(\mathrm{H})$ do not depend on $I_{\mathrm{UV}}$.

\section{Conclusions and discussion}

Models have been presented for inhomogeneous diffuse clouds and spherical clouds. For the former the enhanced penetration of ultraviolet radiation was 


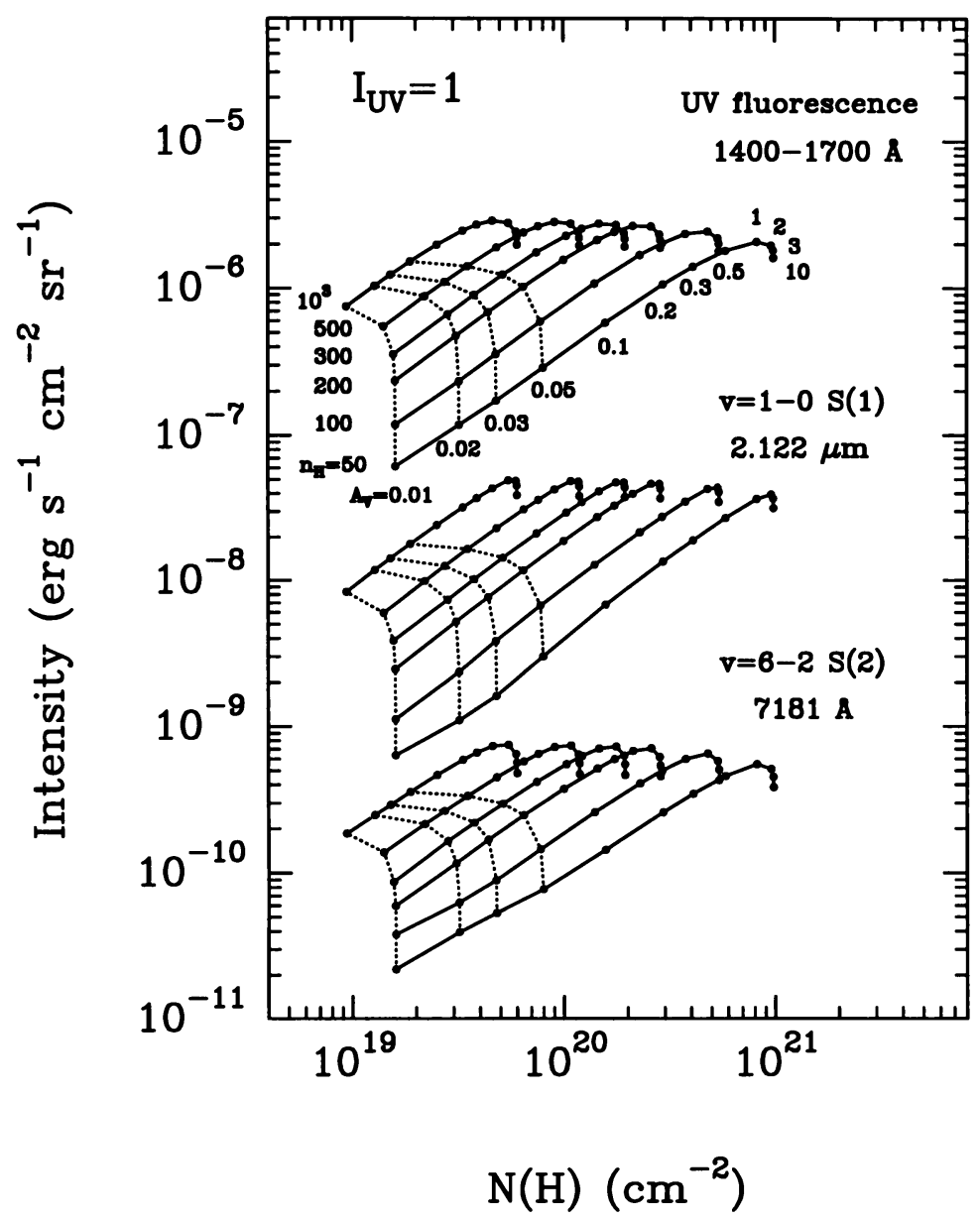

Figure 4. Solid lines: fluorescent line intensities, as functions of atomic hydrogen column density, for several values of the density of $\mathrm{H}$ nuclei. Results apply to the case $I_{U V}=1$ when the incident UV continuum radiation field is equal to that adopted by Draine (1978). Dashed lines connect models with the same total visual extinction across the cloud diameter.

found to yield extended [CII] $158 \mu \mathrm{m}$ and [CI] $609 \mu \mathrm{m}$ emission and strongly enhanced $\mathrm{CO}$ abundances in the shielding clumps. Fluorescently pumped visual and red $\mathrm{H}_{2}$ lines were found to be useful density estimators of the clumps when combined with co-spatial HI observations.

More general, the importance of density inhomogeneities and the consequent need for the determination of their characteristics is apparent. To determine physical parameters more accurately, the density structure and 
geometry of a line of sight needs to be taken into account. The models presented here are a first step in this direction.

\section{References}

Black, J.H., Dalgarno, A., 1977, ApJS 34, 405

Black, J.H., van Dishoeck, E.F., 1987, ApJ 322, 412

Dabrowski, I., 1984, Can. J. Phys 62, 1639

Draine, B.T., 1978, ApJS 36, 595

Falgarone, E., Pérault, M., 1988, A\&A 205, L1

Green S., Truhlar, D.G., 1979, ApJL 231, L101

Howe, J.E., Jaffe, D.T., Genzel, R., Stacey, G.J., 1991, ApJ 373, 158

Marscher, A.P., Moore, E.M., Bania, T.M., 1993, ApJL 419, L101

Meixner, M., Tielens, A.G.G.M., 1993, ApJ 405, 216

Neufeld, D.A., Spaans, M., 1996, ApJ in press

Spaans, M., 1996, A\&A 307, 271

Sternberg, A., 1988, ApJ 332, 400

Stutzki, J., Stacey, G.J., Genzel, R., Harris, A.I., Jaffe, D.T., Lugten, J.B., 1988, ApJ 332,379

Tauber, J.A., Goldsmith, P.F., 1990, ApJL 356, L63

Turner, J., Kirby-Docken, K., Dalgarno, A., 1977, ApJS 35, 281

van Dishoeck, E.F., Black, J.H., 1986, ApJS 62, 109

\section{Discussion}

Pecker: Is not the degree of clumpiness (your clumps seem to have $A_{V} \sim 0.5$ mag) strongly influencing the results? That value should be the largest possible to definitely influence the $\mathrm{CO}$ abundance. Larger $A_{V}$ for individual clumps should lead to $\mathrm{CO}$ values as in the homogeneous case.

Spaans: Yes, the clump size in these models is of the order of $A_{V} \sim 0.5-1 \mathrm{mag}$, right in the regime where $\mathrm{CO}$ becomes self-shielding. For lower extinction clumps, the increase in $\mathrm{CO}$ will not be as large.

Roueff: Assuming $10 \%$ of $\mathrm{C}$ in the form of $\mathrm{PAH}$, what is the fractional abundance of PAH molecules in your model?

Spaans: Approximately $5 \times 10^{-7}$ in the form of $\mathrm{PAH}^{+}, \mathrm{PAH}^{0}$ and $\mathrm{PAH}^{-}$.

Roueff: Previous talks have shown large isotopic ratios $(\geq 100)$ of ${ }^{12} \mathrm{CO} /{ }^{13} \mathrm{CO}$ in diffuse clouds. What is the effect of the clumping on this ratio?

Spaans: Depending on the size of the clumps, $L_{c} \sim 0.02-0.2 \mathrm{pc}$, and the strength of the radiation field $I_{\mathrm{UV}}=1-100$, the ${ }^{12} \mathrm{CO} /{ }^{13} \mathrm{CO}$ ratio varies between 50 and 110. This is mainly caused by the more efficient self-shielding of ${ }^{12} \mathrm{CO}$ versus ${ }^{13} \mathrm{CO}$.

Herbst: Do you have plans to introduce time dependence, such as a finite clump lifetime?

Spaans: In principle, yes. There is no conceptual difficulty with introducing timedependence into the Monte Carlo code besides cpu. Nevertheless, using the correct clump lifetime as a function of its size, density, etc. is a non-trivial problem and one for which detailed hydrodynamic results are required. 